\title{
Research on fault diagnosis system of hall for workshop of meta-synthetic engineering based on information fusion
}

\author{
Guang Yang ${ }^{1 *}$, Shuofeng $\mathrm{Yu}^{2}$ and Shouwen Wen ${ }^{1}$
}

\begin{abstract}
By analyzing multi-sensor information fusion system and hall for workshop of meta-synthetic engineering (HWME) essentially, a universal information fusion system of HWME based on multi-sensor is put forward. Analyzing the fault diagnosis framework of complex system based on information fusion technique, together with the research on the general process of information fusion synthesis fault diagnosis, a fault diagnosis system framework of HWME based on information fusion is set up, which is all-purposed and runs through the whole process of synthetic fault diagnosis. By this method, a new approach to multi-sensor information fusion synthesis fault diagnosis has been found.
\end{abstract}

Keywords: Fault diagnosis, Complex system, HWME, Information fusion

\section{Introduction}

Multi-sensor information fusion technology has been widely applied in the field of fault diagnosis in recent years. Information fusion technology has been applied to fault diagnosis by many scholars, who put forward some diagnostic methods and corresponding diagnostic models according to different problems arising in application. These fusion models can solve a portion of fault diagnosis problems of complex equipment in specific conditions. But the hierarchy, correlativity, time-delay, and uncertainty of the complex system fault make the fault diagnosis difficult. So, there are still some defects and deficiency when single information fusion technology is applied in the field of complex system fault diagnosis system. For example, cutting the organic connection between information due to considering information resource only; just considering the diagnosis of each level instead of the global result; considering just the fusion of various methods without realizing the effectiveness of method fusion. So until now it has not formed a complete, effective, and systematic theoretical framework and

\footnotetext{
*Correspondence: yangguang800122@126.com; sunyoung0783@163.com 'School of Entrepreneurship and Innovation, Shenzhen Polytechnic, Shenzhen, China

Full list of author information is available at the end of the article
}

method in the field of fault diagnosis on multi-source information fusion technology.

Fault-integrated diagnosis system is a complex system to exchange the matter and energy continuously with industrial system, which is influenced deeply by the relations between many aspects in and out of the system. If the process of integrated diagnosis decision is to be scientific, it is unavoidable for it to face complexity and use the idea of complex system to research the diagnosis decision problems. With the rise of the concept of complex system, it has become hot in and out of China to research the complex (adaptive) system and HWME [1, 2]. We analyzed the system, structure, and function of multi-sensor information fusion system and HWME, finding that there are essential similarities between them. In this paper, we do some research on fault-integrated diagnosis system as an open complex system by learning from the research thoughts of HWME with the application of the computer network information technology, complexity science theory, information fusion theory, modern decision theory, and meta-synthesis theory etc., trying to seek a new way to solve the fault diagnosis under complex system, constructing a structure system of 
HWME fault diagnosis based on information fusion to extend and expand the theoretical framework of multi-sensor information fusion fault diagnosis system, improving the ability and the level of diagnosis on complex system on the whole, hoping to provide helpful exploration for setting up a theoretical system framework based on multi-source information fusion fault comprehensive diagnosis.

\section{Technical analysis and research background of systematic HWME based on complexity}

2.1 Research status of HWME based on complexity

With the rapid development of science and technology and the progress of society, more and more complex things and phenomena came into our vision. Scholars and policymakers meet with many essential difficulties when they dealt with these complex problems. One of the important things is that the subjects of modern science and the situation of segmentation make people's identification to the totality and globality of things vague. Facing with more and more complex problems, many researchers start to explore new ways from whole view to solve complex problems. With the above background, "Complexity science" sprouted and grows gradually and has attracted more and more attention of more and more scholars, becoming "A science of the 21st century" [3-5]. In this respect, there are some top representative theories abroad. The theory of self-organization in non-equilibrium systems by Prigogine and Haken in Europe, the theory of complex adaptive system (CAS) by the Santa Fe Institute (SFI) in the USA, and the science of the artificial by Herbert A. Simon [6, 7]. In China, the main one is the open complex giant systems (OCGS) and the research on system complexity of its methodology by Qian Xuesen [8].

\subsection{Technical research of HWME based on complexity}

The meta-syntheses raised by Qian Xuesen bases on the thought of the holism, that is, when they face with complex giant system, people will draw some seeming and sensitive conclusions and judge by experience with the existing theories and their experience, and then integrate, model with these qualitative cognition, make simulation experiment ascend to rational knowledge at last. This will be an iterative process until a conclusion close to the truth is drawn. HWME is a feasible way to make this thought come true. HWME completes the visual process of metasynthesis by using the latest science and technology such as modern information technology, computer network, and artificial intelligence. The core of
HWME is human-computer cooperation (HCC) and meta-synthesis from qualitative to quantitative [9-11].

\subsubsection{Human-computer cooperation (HCC)}

HWME looks human as the components of solution system, making full use of the intelligence of humans. The solution system creates a division on functions and process according to the specialities and advantages of human and machines, forming a human-centered human-computer intelligence system including the advantages of human and machines [3]. In this intelligence system, man and machine use their advantage separately and coordinate well. The ways of human-computer cooperation are human-computer interaction and human-computer collaboration.

Human-computer cooperation is conducive for human to express their opinions and ideas and is also helpful for people to get information, knowledge, and model from the machines. So, the key to design the system is the design of human-machine interface (HMI) and the model of human-machine dialog. The design of human-machine interface (HMI) should consider the availability first because the scholars who are using the system are in wide range. The following three rules should be obeyed: 1) resetting the users under the control; 2) reducing the memory load of users; 3) maintaining consistency in the interface. Human-machine dialog can be applied in many ways to adapt to specific scholars. Intelligent input can be carried out by many technologies such as a stylus, voice, eye-tracking, gesture input, natural language, lip reading, facial recognition, fingerprint and so on with the feels and actions being fully understood. There are various ways for output such as video, audio, graphics as well as text.

Human computer cooperation is the design goal of HWME. Human computer cooperation can make it a reality that humans and computers help each by integrating humans into the system. Both humans and computers have their own advantages. The advantages of humans are embodied in imagery thinking, sudden enlightenment, and experience development, which can play an important role in seminar process controlling, adjusting the parameter of models, generalizing the discussion and selecting schemes. The advantages of computers reflect in the abilities to massively parallel, distribute computing, and manage. So, computers will have an effect that humans cannot match in real-time computing, modeling, emulation, query and so on. The purpose of human-computer collaboration is to combine the qualitative thinking ability of human with the highperformance quantitative calculation ability of the 
machine during the process of dealing with complex problems.

\subsubsection{Meta synthesis from qualitative to quantitative}

Complexity science researches things from the whole first, and then details gradually, synthesizing at last. At the beginning, people's overview to the thing is a solution which is perceived and uncertain. The solution is a result of qualitative analysis, while the symbol of solution is to form a regulative cognition which is a quantitative and can be defined clearly or can be described by models. That means the procedure for people to deal with a problem is a synthesized process from qualitative to quantitative. HWME is an auxiliary tool to solve such problems, which synthesizes the qualitative cognition and information and other knowledge drawn by the scholars by experience through computers and relevant technology, and then model, evaluate, modify for many times, and rise to a global quantitative cognition. This process is very complex and needs to repeat many times. The key technology of HWME is shown in two aspects: one is the meta-synthesis analyzed qualitatively by different scholars, the other is quantitative processing to the final consensus, in other words, it is modeling. If the quantitative conclusion cannot meet the requirements, the research will continue. Thus, we know the process of meta-synthetic is discussion (qualitative analysis) $\rightarrow$ meta synthesis $\rightarrow$ model (processed quantitatively). This is an iterated process as is shown in Fig. 1.

\subsection{The correlational research on this subject}

The author of this paper has dedicated himself to the research on intelligent fault diagnosis area for a long time, especially the research on the theory and method of integrated diagnosis information fusion of charging equipment based on the previous studies of fault diagnosis on charging equipment with the application of multi-resource information fusion technology which developed rapidly in recent years. To the various problems arising in the diagnosis process of industrial equipments, rough set theory, fuzzy theory, neural network, gray theory, genetic algorithm, D-S evidence theory, and the information fusion comprehensive diagnosis theory and method models that are integrated on the theories mentioned above are constructed, respectively. Some valuable research conclusions have been got after the verification by diagnosis on specific equipments. While some factors, such as more and more interrelationships in industrial field, monstrous amount of information, the existence of large-scale systems opened to the outside world bring a problem to be solved urgently. That is how to solve the diagnosis problem of open complex giant systems. Qian Xuesen put forward the thought of open complex giant systems and provided the idea to solve the problems of complex system with HWME. Based on the above background, this paper is trying to construct a universal HWME fault diagnostic system based on the information fusion technology throughout the fault-integrated diagnostic process of complex systems with the essential analysis on information fusion system and HWME, trying to seek a new method of complex system comprehensive fault diagnosis research based on information fusion technology, providing beneficial exploration and reference for sustainable development of information fusion comprehensive diagnostic technology.

\section{Multi-sensor information fusion system framework for HWME}

\subsection{Hierarchy structure of information fusion}

Data level fusion (pixel-level fusion). It is a kind of fusion based on the collected raw data directly. Data is integrated and analyzed before the forecast from the sensors has not been processed. Data level fusion is a low level fusion. The fusion processing is based

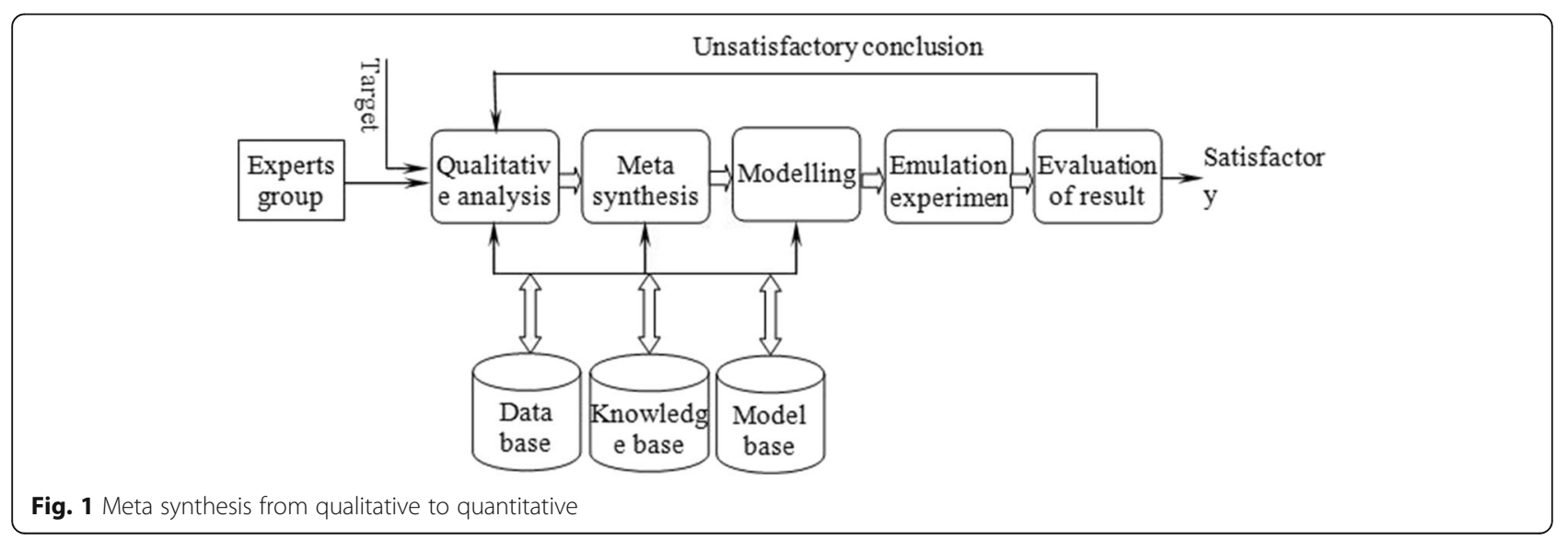


on centralized fusion system. The application of data level fusion is limited because of the matching pixelaccuracy. It is often applied in multi-source image recombination, images analysis and processing, and direct synthesis of the same type of radar waveform.

Feature level fusion. Feature level fusion is to identify feature levels, which is a kind of pattern recognition essentially. Multi-sensor system provides more feature information of relevant objects than single sensor. This enlarges feature space dimension. The concrete way of fusion is the technique relevant to pattern recognition. Correlating features before fusion to classify the feature vectors into meaningful composition. Fault diagnosis occurs mainly in this level.

Decision level fusion. Decision level fusion means to observe the same objective through different types of sensors. Each sensor completes the basic process locally including pre-processing, feature extraction, recognition or decision so as to set up preliminary conclusion on the objective observed, or to make a judge to decision level fusion through correlation processing by utilizing the result of feature level fusion, getting the corollary result at last. Fault diagnosis also occurs in this level.

\subsection{Feature selection and extraction}

Proper feature parameter should be determined before condition monitoring and fault diagnosis as to demonstrate the change of running state of equipments quantitatively. So, it is important to select feature parameters properly. The methods of feature selecting mainly includes neural network selection method, simulated annealing algorithm, Tabu search algorithm, genetic algorithm, optimum parameter searching method and so on.

The more complex the structure of equipment is, the more the types of faults there are. The fault status and features are getting more and more. Feature extraction is necessary in order to make use of the information economically and efficiently. The ordinary methods of feature extraction are principle component feature extraction, feature extraction based on neural network, feature extraction based on wavelet transform, feature extraction based on mutual information entropy, feature extraction based on rough set, feature extraction based on the characteristics of fuzzy information optimization processing, feature extraction based on Euclidean distance, feature extraction based on probability distance measuring, feature extraction based on divergence criterion function, feature extraction based on support vector machine (SVM) etc.

\subsection{The framework of multi-sensor information fusion system based on HWME}

People have applied the technology of multi-sensor information fusion to the research of complex systems at present [12-20], and have set up a common information fusion system of HWME based on multi-sensors by applying the idea of HWME to the research of fault diagnosis complex system, as is shown in Fig. 2.

From Fig. 2, we know that the feature data are extracted from the signal patterns from various sensors,

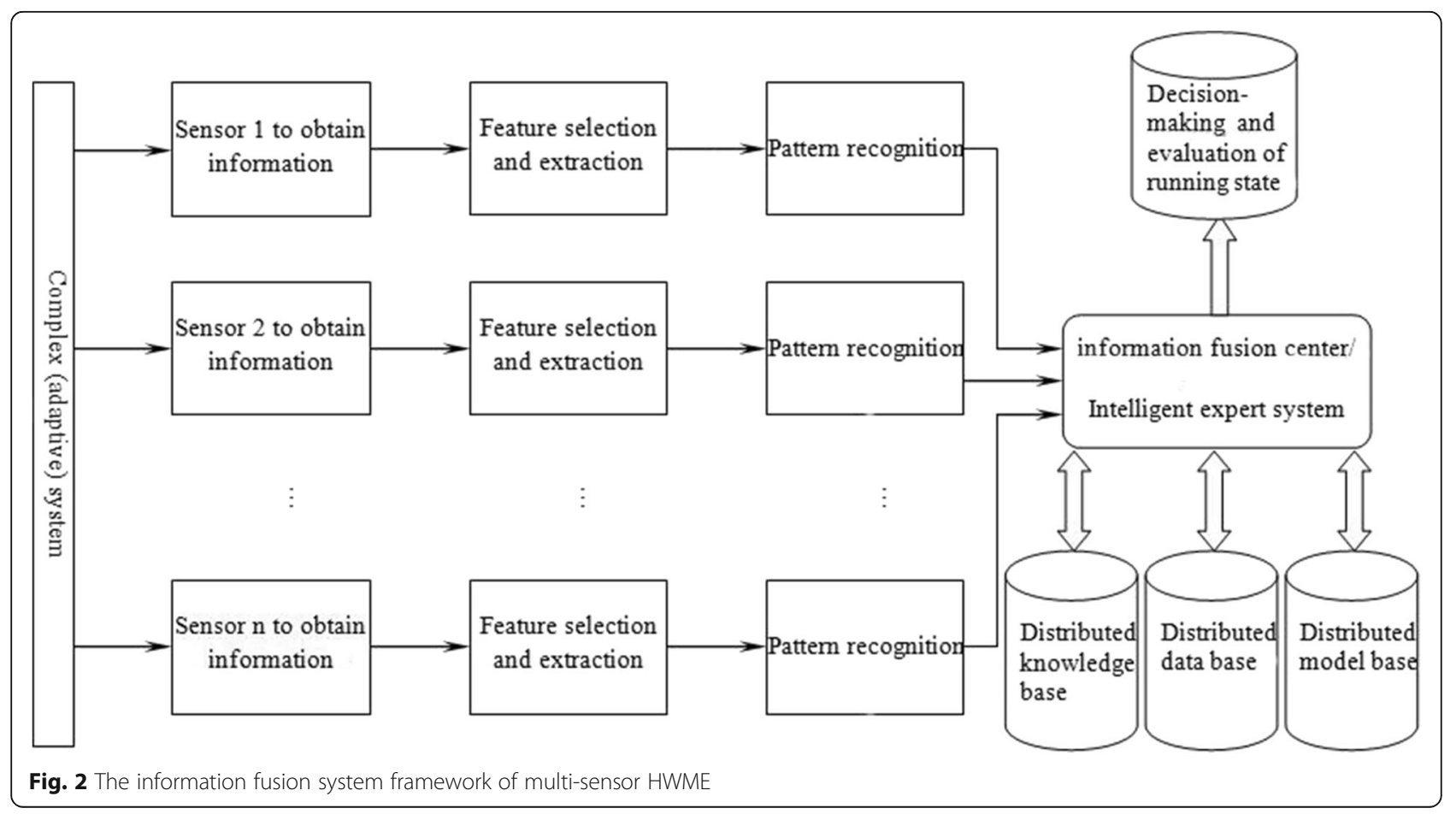


and the extracted feature data are input into the mode discriminator at the same time. Feature level data fusion is done by the mode discriminator to recognize the feature data of system and then input to information fusion center to be decision fusion. When intelligent inference is making in intelligent expert system, special knowledge and rules and parameters relevant will be extracted from distributed knowledge base and data base. They will be matched or fused with feature data and finally make a decision about the basic situation and operation situation of complex (adaptive) system etc.

\section{The design of fault diagnosis system of HWME based on information fusion}

4.1 The structural layer of faulty diagnosis system HWME Considering the open standard system characteristic of HLA (High Level Architecture), the structure of faulty diagnosis system HWME is divided into three layers in this paper according to the thought of OSI/ RM 7-layer structure model, that are high-level faulty diagnosis system hall for workshop, mid-level faulty diagnosis development demonstration hall, and lowlevel faulty diagnosis base resources support hall. In order to build a comprehensive and effective fault diagnosis HWME information fusion system, the technological structure is divided into five layers. The inner structure of faulty diagnosis system HWME can be distinguished into five layers on the technology level, they are network communication layer, base resources layer, integrated support layer, management control layer, and synthetic application layer. All the layers are connected to each other with application program interface API. The structural layers are shown as Fig. 3.

\subsection{The basic framework of HWME comprehensive fault diagnosis system}

Fault diagnosis is a complex system engineering problem [21-25]. Thinking concretely about the problems of the construction and development of comprehensive fault diagnosis system, expert group, data, information relevant and computer simulation are combined together to realize the optimistic construction and sustainable development of fault diagnosis system. This is based on the definition on the basic components and function of the construction and development of fault diagnosis. It also combines the scientific ethics of related discipline and people's experience. Therefore, by taking advantage of the comprehensive system, the HWME of fault diagnosis system from qualitative to quantitative is set up to apply to decision-making and diagnosis scientifically.

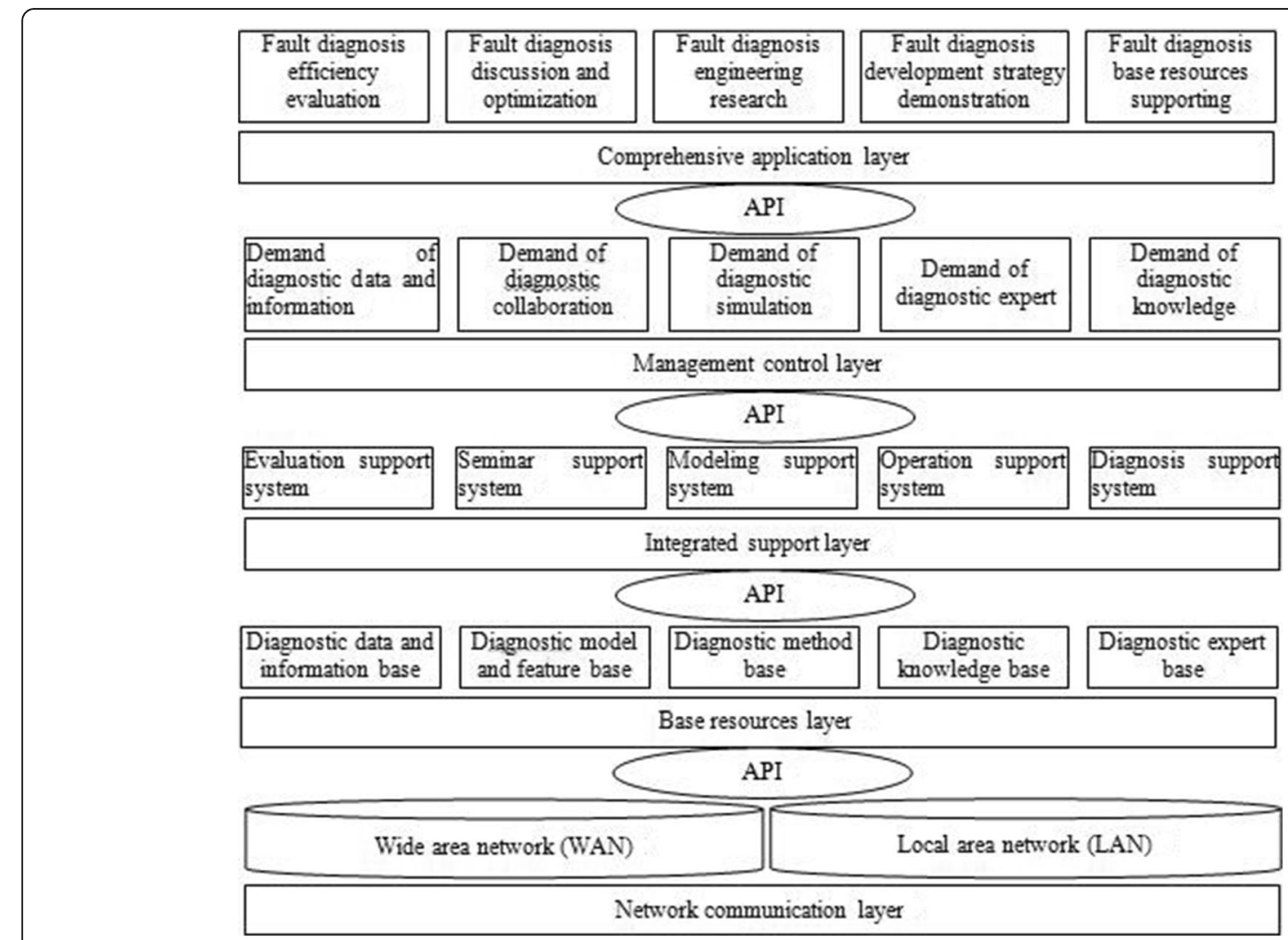

Fig. 3 Structural layer of fault diagnosis system HWME 
By analyzing the function requirement, structure and technology of fault diagnosis HWME information fusion system, combined with the information fusion framework system of multi-sensor HWME as mentioned before, the framework of fault diagnosis system of HWME based on information fusion is set up, as is shown in Fig. 4. From this framework, it is easy to find that the object of HWME is the process of synthesis fault diagnosis. This process can be divided into three levels: fusion diagnosis of data level, fusion diagnosis of feature level and fusion diagnosis of decision level.
The information fusion fault diagnosis HWME is supporting environment in which different diagnostic experts in different fields can work cooperatively. The experts in HWME work cooperatively in a distributed network environment in which the diagnostic experts of various fields can play their own expertise according to their own experience and knowledge with the data and analysis tools from the network; then, they analyze the problems and give opinions. In distributed network environment, different knowledge can be connected together for participants to share, becoming effective. There are more

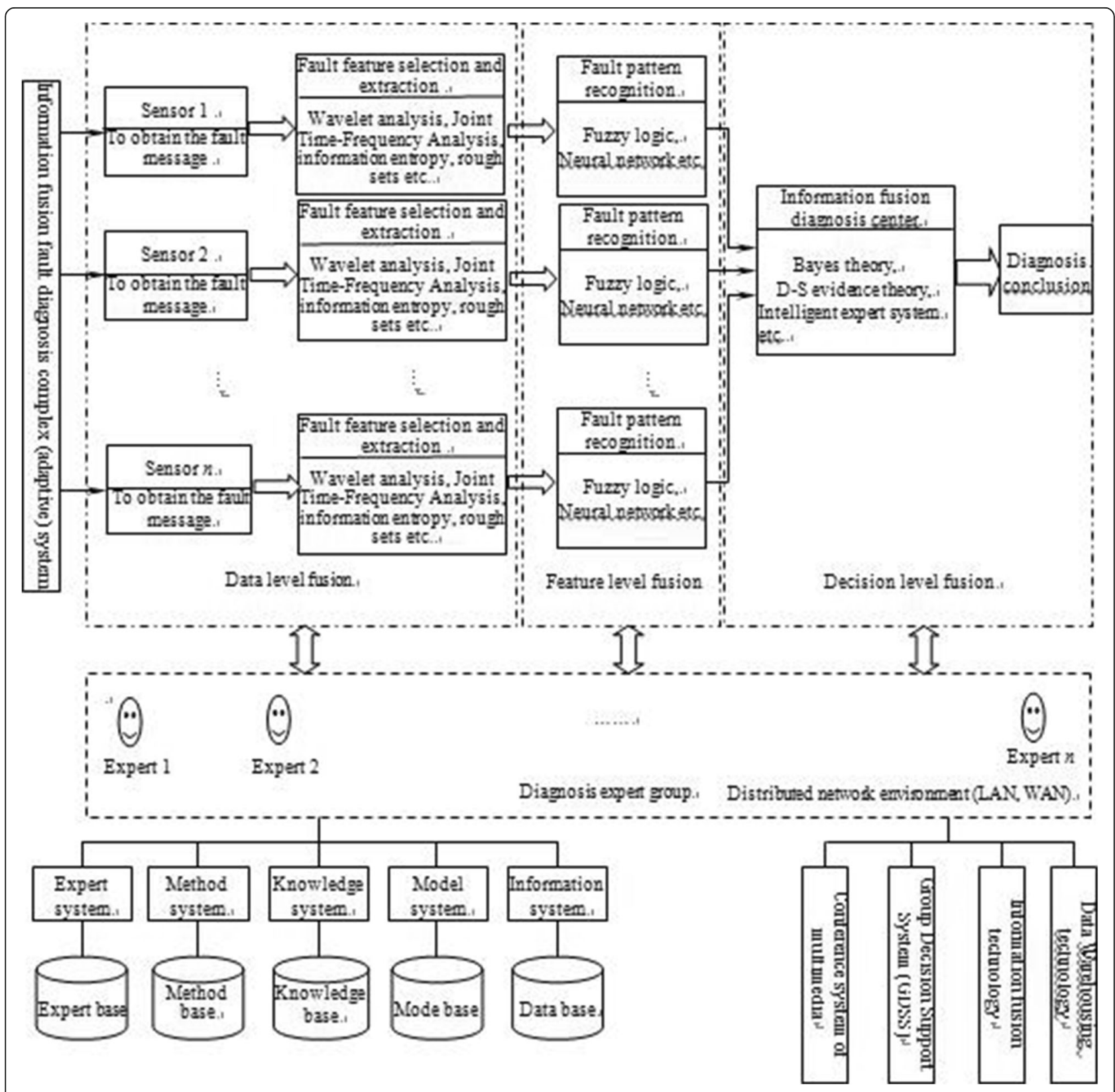

Fig. 4 The structure framework of HWME fault diagnosis system based on information fusion 
than one users, networks, servers, work stations in a complete construction.

4.3 The basic system of synthesis fault diagnosis HWME The system of fault diagnosis HWME consists of five basic systems.

\subsubsection{Expert system}

This system includes diagnostic experts from all kinds of fields, such as machinery diagnostic experts, electronic diagnostic experts, and other diagnostic experts of other fields. The details of each expert are recorded in the expert base including age, sexual, education, specialty, the diagnose projects that they took part in etc. These information can be considered for diagnostic group to select experts and set up expert groups.

\subsubsection{Method system}

Various kinds of methods for diagnostic decision of information fusion are collected and sorted in this system. The information fusion fault diagnostic method base includes fusion diagnostic method based on random models, mainly including Bayesian inference, evidence theory, recursion operator and so on; fusion diagnostic method based on least squares, mainly including Kalman filtering, optimization theory etc.; intelligent fusion diagnostic method mainly including fuzzy logic, neural network, genetic algorithm, gray system, artificial intelligence, rough set theory, support vector machine, wavelet transform and so on; other new meta-synthesis methods developed like wavelet-neural network, fuzzyneural network, fuzzy-gray system and diagnostic method of integrated neural network, etc.

\subsubsection{Knowledge system}

Knowledge system can be divided into three levels from low to high: experience knowledge, science knowledge, and philosophy knowledge. In the information infusion fault diagnosis HWME, the knowledge system emphasized more about the experience knowledge from the diagnostic practice. The knowledge base provides all kinds of diagnosis cases to be considered and referenced and makes a summary about the experience knowledge.

\subsubsection{Model system}

In this system the synthesis diagnostic models of information infusion generally used are collected and sorted. To different diagnostic tasks, the actual situation should be analyzed first before a fault diagnosis is made with the proper diagnostic method and model.

\subsubsection{Information system}

The information system is made of all sorts of data that support the fault diagnosis of information infusion. The data in the data base can be divided into data of equipment types, data of diagnostic experience, data of different kinds of technology and so on.

\subsection{The basic supporting technology of synthesis fault diagnosis system of HWME}

The fault diagnosis HWME based on information infusion is an interdisciplinary and synthesis diagnostic supporting environment including computer technology, decision-making technology, system engineering technology. The rapid development of computer technology, network technology, multi-media technology, and the fast development of VR, artificial intelligence, communication technology provide a good foundation for the creation of fault diagnosis HWME. The fault diagnosis HWME includes four kinds of basic supporting technologies: Multi-media Conference System (MCS), Group Decision Support System (GDSS), information fusion technology, data warehouse technology. In the fault diagnosis HWME, Group Decision Support System integrated Multimedia Conference System (MCS) plays a very important role. The data of Group Decision Support System processed integratedly will be realized through data warehouse technology. Therefore the subject-oriented, summary and collective information can be provided for Group Decision.

Fault Diagnosis System of Hall for Workshop of Metasynthetic Engineering combines the knowledge and intelligence of experts with the technologies of modeling and simulation and AI of computers. It is a "people-based" system integrated with swarm intelligence. It raises the diagnosis judgements from various aspects to scientific conclusion with the advanced technologies of modeling and simulation and AI of computers. With the fault diagnosis system of HWME, domain experts are involved in the diagnosis process with the help of Internet or other communication technology. Therefore more experts can take part in the diagnosis process and the diagnosis time and places are not limited. It makes the diagnosis process democratic and scientific.

\section{Conclusions}

With the development of computer network technology, multimedia technology, information fusion technology and AI, a rapid progress has been made in the field of HWME based on complexity science. HWME has come into truth from assumption. This suggests a new way to set up the HWME fault diagnosis system based on information infusion. The synthesis fault diagnosis process will be scientific, normalized with 
the construction of the fault diagnosis HWME system based on information infusion. The construction of fault diagnosis HWME will provide rich and helpful experience for the construction of workshop hall of fault diagnosis. We should study further to set up a system of fault diagnosis HWME based on information infusion.

(1)We should pay more attention to the accumulation of knowledge and information, including models, methods, experience and perceptual knowledge and so on. We also should collect the experience and theory knowledge from various experts of every field as to set up a complete knowledge system.

(2) We should strengthen the construction of expert base and update the information to realize the dynamic management of expert base.

(3) We should strengthen the construction of information networks because the construction of networks is very important for construction of the fault diagnosis HWME. There will be no HWME without mature construction of networks.

(4) We should emphasize the construction of methods of information infusion as well as the model base, and carry out the research on the methods of synthesis diagnosis of information infusion, especially the research on the synthesis method combining qualitative analysis with quantitative analysis.

\section{Acknowledgements}

The authors acknowledge the National Natural Science Foundation of China (Grant: 60774029) and the National Natural Science Foundation of China (Grant: 71171198)

\section{Authors' contributions}

GY is the corresponding author of the paper. GY is responsible for all aspects of the paper. SY did the part of the experiment. SW also did the part of the experiment. All authors read and approved the final manuscript.

\section{Competing interests}

The authors declare that they have no competing interests.

\section{Publisher's Note}

Springer Nature remains neutral with regard to jurisdictional claims in published maps and institutional affiliations.

\begin{abstract}
Author details
${ }^{1}$ School of Entrepreneurship and Innovation, Shenzhen Polytechnic, Shenzhen, China. ${ }^{2}$ School of Applied Foreign Language, Shenzhen Polytechnic, Shenzhen, China.
\end{abstract}

Received: 16 October 2017 Accepted: 13 December 2017

Published online: 29 December 2017

\section{References}

1. Dai, R. (2011). From engineering cybernetics to information space HWME-the innovation and development of system science. J. Univ. Shanghai Sci. Technol., 06, 543-547+508.

2. Yin, H, \& Dai, R. (2011). Noetic and intelligence sciences and engineering. Univ. Shanghai Sci. Technol., 01, 18-23.
3. Dai, R. (2011). Formation and development of social intelligence science. Univ. Shanghai Sci. Technol., 01, 1-7.

4. Dai, R, \& Li, YD. (2004). Researches on hall for workshop of meta synthetic engineering and system complexity. Compl. Syst. Compl. Sci., 1(4), 1-24.

5. Waldrop, MM (1992). Complexity: the emerging science at the edge of order and chaos. New York: Simon \& Schuster.

6. Horgan, J. (1995). From complexity to perplexity. Sci. Am., 272(6), 74-79.

7. Simon, HA (2004). The sciences of the artificial — analyzing complexity and complex systems. Translated by Wu Yishan. Shanghai: Shanghai Technology Education Press.

8. Zheng, N, \& Dai, R. (2014). Mr. Qian xuesen's academic thought of "metasynthetic wisdom" of the cutting-edge. Control Theory Appl., 12, 1606-1609.

9. Dai, R, Li, Y, Li, Q. (2012). Meta synthesis opens the light of wisdom. Autom. Panorama, 02, 10-14.

10. Pasello, M, Zamboni, S, Mallano, A, Flego, M, Picci, P, Cianfriglia, M, Scotlandi, K. (2016). Design and construction of a new human Naïve single-chain fragment variable antibody library, IORISS1. J. Biotechnol., 224, 1-11.

11. Cao, Y, Xue, H, Wan, Y, Xu, Y. (2017). Decision support system for solution to initial water rights allocation based on HWMSE. Value Eng., 15, 62-64.

12. Yu, B. (2016). Complex system education decision under big data technology - the application of HWME. China Manag. Informationization, 19(21), 231-233.

13. Yu, B. (2016). The study of the education decision-making: based on the hall for workshop of metasynthetic engineering. Value Eng., 34, 20-23.

14. Song, X, Wang, G, Feng, X. (2006). Research on fault diagnosis of complex system based on information fusion technology. Chin. J. Sci. Instrum., 27(6), $1764-1765+1772$

15. Ma, A. (2014). HWME—an intelligent solving method of complex giant systems based on the scientific thought of Qian Xuesen. Mark. Modernization, 12, 50.

16. Li, J, Wang, H, Xie, J, Zhang, Y. (2012). Construction of complex water resources allocation system based on HWME. J. Water Resour. Water Eng., 23(1), 22-25+29.

17. $\mathrm{Wu}, \mathrm{L}$, Pan, G, Huang, Q. (2014). Method of multiple sources information fusion for complex system with small sample test based on ML-Il theory. Comput. Eng. Appl., 50(15), 220-222.

18. Shang, F, Yao, A, Fu, P. (2011). The HWME system and key technology of ship power plant design. China new technol. \& products, 17, 25-26.

19. ZhongLiang, J, Han, P, YanYuan, Q. (2013). Current progress of information fusion in China. Chin. Sci. Bull., 58(36), 4533-4540.

20. LV, R, \& Sun, L. (2017). Fault diagnosis method of complex system based on multi-source information fusion fault tree and fuzzy Petri net. Comput. Integr. Manuf. Syst., 23(8), 1817-1831.

21. Cao, S, Han, Y, Sun, X. (2009). Research on process design of intelligent fault diagnosis for complicated system. Mach. Des. Manuf., 4, 60-62.

22. Ma, J, LV, C, Liu, H. (2010). Study on two key techniques of complex system fault diagnosis. J. Test Meas. Technol., 24(4), 372-376.

23. Zhao, Y, Dong, C, Zhang, Q. (2016). Fault diagnostics using DUCG in complex systems. J. Tsinghua Univ. (Sci. Technol.), 56(5), 530-537+543.

24. Wang, G, Li, Y, Song, H, Li, Y. (2014). Study on improving Bayesian networkbased fault diagnosis technology. Coal Chem. Ind., 37(11), 4-7.

25. Ge, Z. (2017). Research on fault diagnosis of complex system. Ind. Sci. Tribune, 16(11), 79-80.

\section{Submit your manuscript to a SpringerOpen ${ }^{\circ}$ journal and benefit from:}

- Convenient online submission

Rigorous peer review

- Open access: articles freely available online

- High visibility within the field

- Retaining the copyright to your article

Submit your next manuscript at $>$ springeropen.com 